\title{
Characteristics of Calls to a COVID-19 Mental Health Hotline in the First Wave of the Pandemic in New York
}

\author{
Hussain Abdullah ${ }^{1} \cdot$ Sean Lynch ${ }^{2} \cdot$ Sahar Aftab $^{3} \cdot$ Sivan Shahar $^{2} \cdot$ Lidia Klepacz $^{1,2} \cdot$ Paul Cristofano $^{1} \cdot$ \\ Saad Rahmat ${ }^{4} \cdot$ Jaya Save-Mundra' ${ }^{1}$ Rhea Dornbush ${ }^{1,2} \cdot$ Alexander Lerman $^{1,2} \cdot$ Karen Berger $^{1} \cdot$ Abraham Bartell $^{1,2}$. \\ Stephen J. Ferrando ${ }^{1,2}$
}

Received: 13 March 2021 / Accepted: 27 June 2021 / Published online: 10 July 2021

(c) The Author(s), under exclusive licence to Springer Science+Business Media, LLC, part of Springer Nature 2021

\begin{abstract}
This report describes the development, implementation and outcomes of a "COVID-19 Anxiety Hotline," designed to address the community's mental health crisis provoked by the coronavirus pandemic. The service was specifically designed using survey data regarding the effects of the COVID-19 pandemic on its staff and community members. Callers had aroundthe-clock direct access to mental healthcare providers at no cost. Quantitative analysis showed that nearly three out of four callers experienced new onset anxiety and insomnia driven by fear of exposure, and had difficulty accessing mental health care. In addition to immediate support, referral to tele-mental health care was provided to $86 \%$ of callers. Qualitative analysis indicates the effectiveness of immediate support and appropriate referrals using a tele-health platform. Our report indicates that the service was utilized by the general population, by health care workers, and rapidly provided referrals to individuals with limited access to mental health care during the pandemic.
\end{abstract}

Keywords COVID-19 $\cdot$ Psychological distress $\cdot$ Mental health resources $\cdot$ Depression $\cdot$ Anxiety $\cdot$ Tele-health

\section{Introduction}

Coronavirus 2019 disease (COVID-19) is caused by novel severe acute respiratory syndrome coronavirus-2 (SARSCoV-2) with over 450,000 deaths reported so far in the United States (Bilinski \& Emmanuel, 2020). The stress of the pandemic and the viral infection itself produced a host of adverse mental health and neuropsychiatric effects (Ferrando et al., 2020). A U.S. general population survey conducted during the First Wave of the pandemic indicated that $33 \%$ of the population endorsed mental health

Stephen J. Ferrando

Stephen.Ferrando@WMCHealth.org

1 Department of Psychiatry, Westchester Medical Center Health Network, 100 Woods Road, Valhalla, NY 10595, USA

2 Department of Psychiatry and Behavioral Sciences, New York Medical College, Valhalla, USA

3 Department of Emergency Medicine, Vassar Brothers Medical Center, Poughkeepsie, USA

4 Department of Psychiatry and Behavioral Sciences, Boston Children's Hospital, Harvard Medical School, Boston, USA symptoms, particularly depression and anxiety, increased from a base rate of $19 \%$ prior to the pandemic (Pierce et al., 2020). Mental Health America online screenings increased $400 \%$, with an increase in depression, anxiety, suicidal ideation and psychosis (Yasgur, 2020). Health care workers and first responders are at particular risk: 11-73\% of physicians, nurses, and auxiliary staff report post-traumatic stress symptoms; $27.5-50.7 \%$ reported depressive symptoms; $34-36.1 \%$ reported insomnia symptoms; and 45\% reported severe anxiety symptoms (Preti et al., 2020). A higher proportion of adult patients presenting for emergency department psychiatric evaluations during First Wave of the pandemic did not have outpatient care or could not contact their outpatient providers compared to a similar period prior to the pandemic (Ferrando et al., 2020). Thus, innovative interventions, such as the widespread use of tele-mental health platforms with direct access to mental healthcare providers, have been employed to overcome some of these pandemicrelated barriers. 


\section{Methods}

Here we describe the development, implementation and outcomes of a "COVID Anxiety Hotline" that the Department of Psychiatry at Westchester Medical Center Health Network (WMCHealth) in Valhalla, New York launched in April 2020 to address the need for immediate mental health support for WMCHealth staff and the surrounding community. This service was operated through a WMCHealth call-in center for on-site COVID-19 testing that allowed individuals to connect immediately with psychiatry residents and psychologists, at any hour of the day at no cost. Data derived from an internal survey of our own staff indicated high rates of stress and anxiety, fear of contracting COVID-19, and of infecting family members. We utilized this information to develop a set of support and coping interventions designed to: (1) de-escalate acute distress using supportive listening and mindfulness techniques, (2) provide factual information about COVID-19 risk and prevention, (3) enhance active coping skills, and (4) provide referrals to mental health treatment resources, including our own outpatient department.

Hotline staff trained in the interventions were provided with written guidelines and referral resources. Staff collected information about each call, including demographics, presenting problem, psychiatric history, level of acuity, and whether or not a mental health referral was made. Acuity was considered low when the presenting symptoms and problem could be managed during a relatively brief phone intervention and necessitated either no follow-up or a non-urgent referral to outpatient care. Acuity level was defined as high when the presenting symptoms and problem required intensive intervention such as next-day outpatient appointments or rapid referrals to psychiatric emergency services. Hotline staff had attending psychiatry staff backup at all times in the event of any difficult clinical scenarios.

For this report, we analyzed hotline calls between April 10, 2020 and July 1, 2020. A total of 177 calls regarding acute mental health symptoms and/or inquiries related to COVID-19 were included in the analysis. Group differences were measured using Pearson Chi Square tests. All the tests were twotailed with $5 \%$ level of significance. The data was analyzed using SPSS version 18.0 (SPSS Inc. Chicago IL, United States, 2009). This study was approved by the Institutional Review Board (IRB) of New York Medical College and the Westchester Medical Center Clinical Research Institute. The authors certify responsibility for this study. There are no known associated conflicts of interest.

\section{Results}

The majority of the callers were females $(80.6 \%)$, and $15.3 \%$ of calls were from healthcare workers. $58.3 \%$ of callers did not report any prior psychiatric history, while $22.2 \%$ confirmed a history of anxiety. Anxiety and sleep disturbances were the most prevalent symptoms reported by callers $(72.2 \%)$. The most common reason overall for the experienced symptoms was reported to be fear of COVID19 exposure $(52.8 \%)$. More than $50 \%$ of callers presenting with anxiety and insomnia indicated that their symptoms were secondary to fear of COVID-19 exposure ( $\mathrm{p} \leq 0.05)$. Data reveals $26.4 \%$ of calls were of high acuity level, which was significantly associated with insomnia and anxiety $(\mathrm{p} \leq 0.05)$. Hotline staff provided referrals for mental health care to $86.2 \%$ of callers. All of the callers reporting history of anxiety and depression received mental health care referrals, while $51.6 \%$ of the callers with no prior psychiatric history received mental health care referral $(\mathrm{p} \leq 0.05)$.

There were 5 individuals who accounted for a total of 105 repeat calls. All 5 callers had a prior psychiatric and/ or substance use history, and had experienced difficulty accessing care due to quarantine restrictions and scarce mental health resources. One caller, Mr. W, was an elderly man with a history of alcohol use disorder, who called 27 times. He reported increased alcohol use during the pandemic in the context of isolation, limited social support, and stress imposed by the lockdown. His older sister was admitted to the hospital for COVID-19, and he expressed concerns regarding her health. Over the course of multiple calls, he was provided with supportive listening, calming techniques, and motivational interviewing to accept help for his alcohol use and anxiety. Eventually, he consented to tele-health treatment at our outpatient psychiatry clinic.

Mr. Y was a young man who called 35 times. He was previously enrolled in an outpatient partial hospitalization program which was taken offline due to the pandemic. He reported stress and anxiety in the context of losing contact with his providers, as well as frequent arguments with family members at home due to social isolation and quarantine precautions. The Hotline became a de-facto bridge treatment for him, providing supportive contact, and calming and de-escalation techniques until outpatient tele-mental health could be arranged.

Health care workers also benefited from the Hotline. For example, a registered nurse called for support in the setting of new-onset severe panic attacks due to the intensity of her exposure to critically ill patients with COVID-19, particularly patients who died but were unable to see their families. She was referred for a crisis evaluation at the Emergency Department and was immediately linked to the mental health clinic for psychotherapy and medication management. 


\section{Discussion}

The COVID-19 pandemic has significantly impacted the mental health of the general population, and has been especially detrimental to those individuals at higher risk, including individuals directly affected by COVID-19, those with existing physical/psychological morbidities, and health care workers (Rajkumar, 2020). There have been increasing reports of significant worsening of pre-existing mental health issues in addition to the new onset of anxiety, depression, and other psychiatric conditions (Rajkumar, 2020). Furthermore, COVID-19 related stressors, including but not limited to, isolation, health-related concerns, and the financial impact of the pandemic, have all been associated with psychiatric symptoms (Ferrando et al., 2020), and are still just as prevalent now as they were in March 2020.

Our findings emphasize that the role of mental health specialists is crucial during the current pandemic and stresses the importance of being proactive in addressing the needs of our communities. Our earlier survey results (Ferrando et al., 2020) are consistent with the current report of severe limitations experienced by patients in accessing mental health care during the pandemic.

Based on the results obtained, the COVID-19 Mental Health Hotline appears to have successfully targeted individuals in both the health care setting and in the general population. The number and variety of calls suggests that the service was utilized well by the target populations, which was likely due to the rapid implementation of the hotline. It also successfully served a small subset of the population that required multiple calls and who utilized the service as a method of transitional care at no cost. Moreover, this unique service appears to be a good allocation of resources during the ongoing pandemic to enhance real-time access to mental health care services and to facilitate mental health referrals. As the pandemic continues to cause worldwide distress, mental health services must be adequately staffed and prepared to screen and treat those individuals who need our assistance. The implementation of a mental health hotline service has been a relatively simple and cost-effective manner to provide access to services which otherwise have been very difficult to obtain during this global crisis.

This study was limited due to the nature of the hotline calls, which were not formal clinical encounters, and therefore possesses a limited clinical framework for the evaluation of symptoms. Despite this limitation, we believe that the framework of our Hotline service could be successfully adapted by others, and that institutions across the country should further develop easily accessible tele-mental health services at no cost to minimize Emergency Department visits and appropriately triage psychiatric care based on acuity.
COVID-19 Mental Health Hotline for their participation and support throughout this study. The COVID-19 Mental Health Hotline was sponsored by the Department of Psychiatry at Westchester Medical Center Health Network. This service was utilized as a supportive resource by Westchester Medical Center Health staff and the Westchester community at large.

Author Contribution Authors of this manuscript contributed to the implementation and design of this study, data collection and analysis, and development and review of the manuscript.

Funding Funding for the COVID-19 Mental Health Hotline was provided by WMCHealth and New York Medical College. No funds were required for the completion of this study.

Data Availability The data was analyzed using SPSS version 18.0 (SPSS Inc. Chicago IL, United States, 2009).

\section{Declarations}

Conflict of interest The authors declare that they have no conflicts of interest.

Ethical Approval This research study was completed in accordance with the ethical standards described in the 1964 Declaration of Helsinki and was approved by the New York Medical College Institutional Review Board and the Westchester Medical Center Clinical Research Institute.

\section{References}

Bilinski, A., \& Emanuel, E. J. (2020). COVID-19 and excess allcause mortality in the US and 18 Comparison Countries. JAMA, 324(20), 2100-2102. https://doi.org/10.1001/jama.2020.20717

Ferrando, S., Klepacz, L., Lynch, S., Shahar, S., Tavakkoli, M., Dornbush, R., \& Bartell, A. (2020). Psychiatric emergencies during the height of the COVID-19 Pandemic in the Suburban New York City Area. Journal of Psychiatric Research. https://doi.org/10. 1016/j.jpsychires.2020.10.029

Pierce, M., Hope, H., Ford, T., Hatch, S., Hotopf, M., John, A., et al. (2020). Mental health before and during the COVID-19 pandemic: A longitudinal probability sample survey of the UK population. The Lancet Psychiatry. https://doi.org/10.1016/S2215-0366(20) 30308-4

Preti, E., Di Mattei, V., Perego, G., Ferrari, F., Mazetti, M., Taranto, P., Di Pierro, R., Madeddu, F., \& Calati, R. (2020). The psychological impact of epidemic and pandemic outbreaks on healthcare workers: Rapid review of the evidence. Current Psychiatry Reports, 22(8), 43. https://doi.org/10.1007/s11920-020-01166-z

Rajkumar, R. P. (2020). COVID-19 and mental health: A review of the existing literature. Asian Journal of Psychiatry., 52, 102066. https://doi.org/10.1016/j.ajp.2020.102066

Yasgur, B.S. (2020). Staggering increase in COVID-linked depression, anxiety. Medscape. Retrieved from https://www.medscape.com/ viewarticle/934882.

Publisher's Note Springer Nature remains neutral with regard to jurisdictional claims in published maps and institutional affiliations.
Acknowledgements The authors would like to thank Westchester Medical Center, New York Medical College, and all of the users of the 